\title{
FREQUÊNCIA DE CASOS DE MORTALIDADE INFANTIL NO ESTADO DE PERNAMBUCO NO PERÍODO DE 2013 A 2017
}

\author{
NUMBER OF CASES OF CHILD MORTALITY IN THE STATE OF \\ PERNAMBUCO IN THE PERIOD 2013 TO 2017
}

\author{
Dáfiny do Nascimento Costa ${ }^{1}$ \\ Kathia Rayane dos Santos Lima ${ }^{2}$ \\ Lívia Mirelly Ferreira de Lima ${ }^{3}$ \\ Amanda Tavares Xavier ${ }^{4}$
}

RESUMO: OBJETIVO: Identificar a frequência de casos de mortalidade infantil no Estado de Pernambuco no período de 2013 a 2017. MÉTODO: Trata-se de um estudo quantitativo, descritivo e observacional. Os dados foram coletados no Departamento de Informática do Sistema Único de Saúde (DATASUS) a partir do Sistema de Informação de Mortalidade - SIM. RESULTADOS: Foram registrados 9.246 óbitos infantis no estado de Pernambuco no período de 2013 a 2017, desses $54,4 \%$ são do sexo masculino. O período neonatal precoce é a fase que mais ocorrem os óbitos, representando mais da metade dos casos dos óbitos infantis. Em relação aos dados maternos, 22,8\% das mães eram adultas jovem e 51,3\% com escolaridade acima de oito anos de estudo. As principais causas dos óbitos estão ligados ao período perinatal $(57,2 \%)$. CONCLUSÃO: Ressaltamos a importância da ampliação na assistência adequada durante todo esse período, pré-natal, parto, nascimento e valorização do planejamento reprodutivo, do fortalecimento da atenção primária à saúde.

Palavras chave: Enfermagem em saúde pública; Mortalidade Infantil; Vigilância epidemiologia.

\footnotetext{
${ }^{1}$ Acadêmica do curso de Enfermagem da Univisa, Centro Universitário da Vitória de Santo Antão. Vitória de Santo Antão, PE, Brasil.

${ }^{2}$ Acadêmica do curso de Enfermagem da Univisa, Centro Universitário da Vitória de Santo Antão. Vitória de Santo Antão, PE, Brasil.

${ }^{3}$ Enfermeira pela Univisa, Centro Universitário da Vitória de Santo Antão. Coordenadora de Vigilância Epidemiológica Hospitalar no município de Vitória de Santo Antão. Vitória de Santo Antão, PE, Brasil.

${ }^{4}$ Enfermeira. Docente do Centro Universitário da Vitória de Santo Antão (UNIVISA). Vitória de Santo Antão, PE, Brasil.
} 
ABSTRACT: OBJECTIVE: To identify the frequency of cases of infant mortality in the State of Pernambuco from 2013 to 2017. METHOD: This is a quantitative, descriptive and observational study. Data were collected at the Informatics Department of the Unified Health System (DATASUS) from the Mortality Information System - SIM. RESULTS: 9,246 infant deaths were recorded in the state of Pernambuco from 2013 to 2017, of which 54.4\% are male. The early neonatal period is the stage that most deaths occur, representing more than half of the cases of infant deaths. Regarding maternal data, $22.8 \%$ of mothers were young adults and $51.3 \%$ had schooling over eight years of study. The main causes of death are linked to the perinatal period (57.2\%). CONCLUSION: We emphasize the importance of expanding appropriate care throughout this period, prenatal, childbirth, birth and enhancement of reproductive planning, strengthening of primary health care.

Keywords: Public Health Nursing; Child Mortality; Epidemiology Surveillance. 


\section{INTRODUÇÃO}

No Brasil, em 2017, ocorreram 36.223 óbitos no período da infância. A mortalidade infantil refere-se aos óbitos menores de 1 ano de vida, sendo subdividida em mortalidade neonatal (0-27 dias de vida) e pós-neonatal (28 dias até 364 dias de vida). A mortalidade neonatal é dividida ainda em neonatal precoce ( 0 a 6 dias de vida) e neonatal tardia (de 7 a 27 dias de vida) (MS, 2017).

$O$ coeficiente de mortalidade infantil é considerado um indicador de grande valor para a saúde, por refletir as condições da assistência ao pré-natal, parto e puerpério. A Organização Mundial de Saúde (OMS), em 2010, lançou uma normativa com uma política de investigação de óbito infantil por Estado, a partir da experiência de alguns municípios que desenvolviam essa estratégia (OLIVEIRA, et al. 2018).

Segundo Leal, há um decréscimo relevante no coeficiente de mortalidade do Brasil, porém ainda há desigualdade relacionadas ao desenvolvimento socioeconômico e ao acesso a serviços de saúde, que precisam ser superadas, além de identificação dos fatores associados a mortalidade infantil, $70 \%$ dos óbitos neonatais poderiam ser evitados se fossem prestadas um cuidado adequado a gestante e ao recém-nascido, por esse motivo é essencial a identificação dos fatores associado a esses óbitos (MIGOTO, et al. 2018).

Entre os óbitos que poderiam ter sido evitados, destacou-se como causa os transtornos respiratórios e cardiovasculares, infecção especifica do período perinatal, transtornos relacionados com a duração da gestação e crescimento fetal, tudo isso com uma assistência adequada não ocorriam esses tipos de óbitos neonatais com essas causas (FILHO, et al. 2017). No estudo realizado a partir de uma análise das ações do Sistema Único de Saúde no Brasil, observou-se que uma assistência prestada ao parto reduziu nesse período, causas como hipóxia intrauterina, a asfixia ao nascer e a aspiração neonatal tiveram redução importante. 
Houve redução dos óbitos por transtornos respiratórios específicos e por infecções especificas no período neonatal, exceto rubéola e hepatite viral congênita (MALTA, et al., 2019).

Diante do exposto se faz necessário a realização de estudos epidemiológicos que analisem a mortalidade infantil no país. Os achados provenientes desses estudos poderão servir de subsídios para entendimento do cenário existente sobre a temática e a partir disso, melhorar as ações direcionadas ao controle dos casos, melhora dos indicadores e da assistência de saúde desde o pré-natal até o puerpério.

\section{OBJETIVO}

Identificar o número de casos de mortalidade infantil no Estado de Pernambuco no período de 2013 a 2017.

\section{MÉTODO}

Trata-se de um estudo quantitativo, descritivo, observacional, cujos dados foram coletados no Departamento de Informático do Sistema Único de Saúde (DATASUS), através do Sistema de Informação de Mortalidade - SIM. Mediu-se a proporção e o perfil dos casos de mortalidade infantil.

O instrumento preenchido para confirmação do óbito é a DO (Declaração de Óbito). Após seu preenchimento, as informações são digitadas no SIM e a Secretaria do Estado encaminha os dados para o Ministério da Saúde, que publica no DATASUS. Os dados são então tabulados no sistema Tabnet e fica disponibilizado de forma online.

Foram utilizados como critérios de inclusão, todos os dados coletados, que apontaram um percentual considerável de completude, ou seja, maior que $80 \%$, para 
gerar uma informação fidedigna. Já como critério de exclusão foram usados os indicadores que possuíam mais de $20 \%$ de respostas ignoradas ou em branco e que estavam fora do objetivo do estudo.

Os dados foram organizados em tabelas elaboradas empregando-se o software Microsoft Excel 2010, produzido pela Microsoft Corporation. Como produto, as análises foram distribuídas em frequências absolutas e analisadas através de percentual simples.

\section{RESULTADOS}

Foram registrados 9.246 óbitos infantis no estado de Pernambuco no período de 2013 a 2017 , desses $54,4 \%$ são do sexo masculino, o período neonatal precoce é a fase que mais ocorrem os óbitos, sendo mais da metade dos óbitos infantis. Destacam-se na faixa etária da mãe, o período do adulto jovem. Escolaridade acima de oito anos de estudo (Tabela 1). 
Tabela 1: Perfil epidemiológico dos óbitos infantis ocorridos no Estado de Pernambuco no período de 2013 a 2017.

\begin{tabular}{cccc}
\hline Variáveis & Categorias & $\mathbf{N}^{*}$ & $\%$ \\
\hline \multirow{4}{*}{ Sexo } & Masculino & 5.032 & 54,4 \\
& Feminino & 4.121 & 44,6 \\
& Ignorado & 93 & 1,0 \\
\hline & Neonatal precoce & 4.997 & 54,0 \\
& Neonatal tardio & 1.513 & 16,4 \\
& Pós neonatal & 2.734 & 29,6 \\
& Ignorado & 2 & 0,0 \\
\hline \multirow{5}{*}{ Faixa etária } & $10-14$ & 148 & 1,6 \\
& $15-19$ & 1.884 & 20,4 \\
& $20-24$ & 2.108 & 22,8 \\
& $25-29$ & 1.796 & 19,4 \\
& $30-34$ & 1.321 & 14,3 \\
& $35-39$ & 803 & 8,7 \\
& $40-44$ & 340 & 3,7 \\
& $45-49$ & 32 & 0,3 \\
& $50-54$ & 1 & 0,0 \\
& Idade ignorada & 813 & 8,8 \\
\hline \multirow{5}{*}{ Escolaridade da mãe } & Nenhuma & 367 & 4,0 \\
& 1 a 3 anos & 717 & 7,8 \\
& 4 a 7 anos & 2.393 & 25,9 \\
& 8 a 11 anos & 3.889 & 42,1 \\
Total & 12 anos e mais & 848 & 9,2 \\
\cline { 2 - 4 } & Ignorado & 1.032 & 11,2 \\
\cline { 2 - 4 } & & $\mathbf{9 . 2 4 6}$ & $\mathbf{1 0 0 , 0}$ \\
\hline
\end{tabular}

Fonte: MS/SVS/CGIAE - Sistema de Informações sobre Mortalidade - SIM / 20132017. *Número de Casos.

O gráfico 1 abaixo mostra uma redução no número de mortalidade infantil, no decorrer dos anos analisados. 
Gráfico 1: Número de óbitos infantis segundo o ano de ocorrência no Estado de Pernambuco.

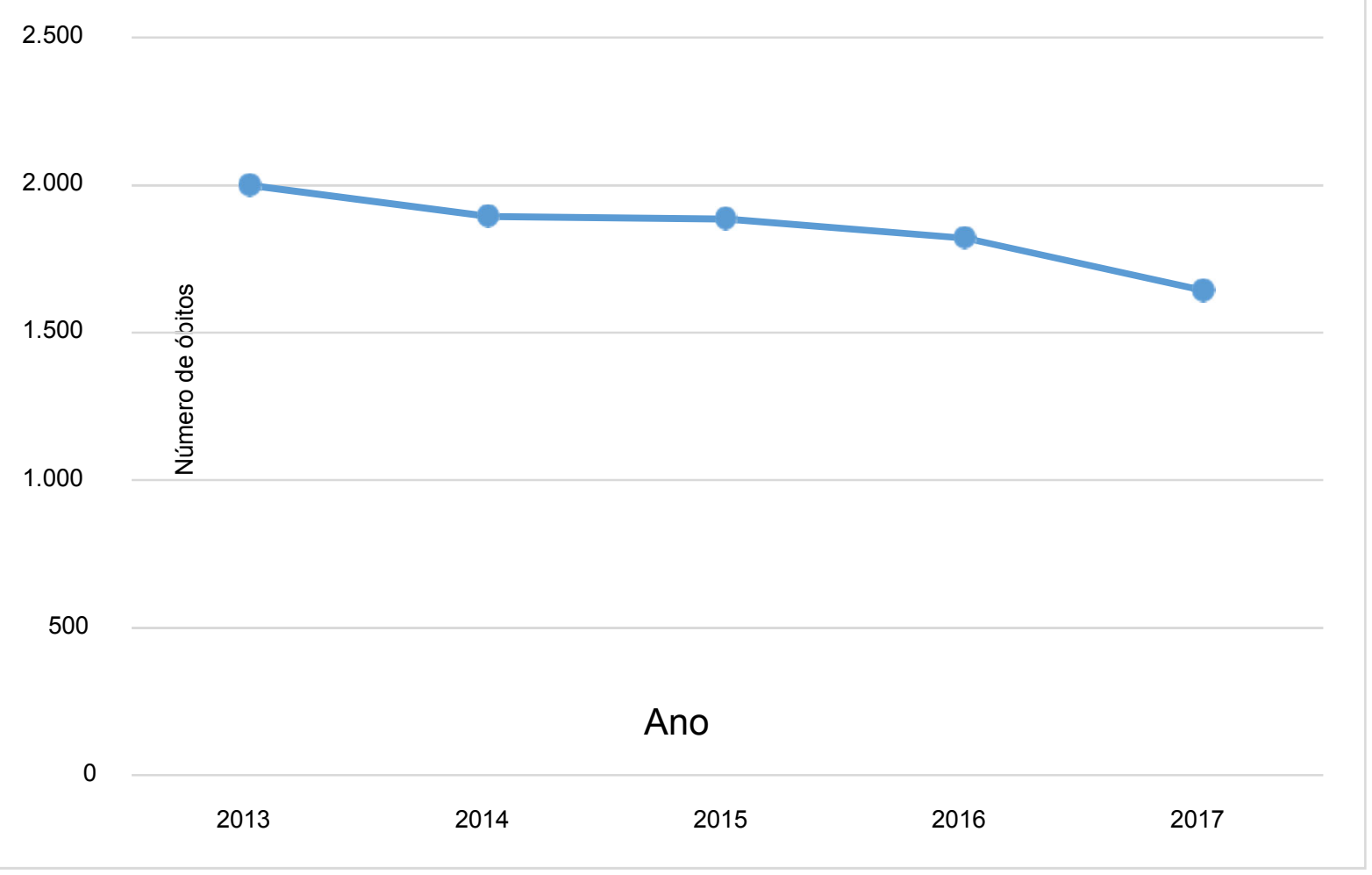

Fonte: MS/SVS/CGIAE - Sistema de Informações sobre Mortalidade - SIM / 20132017.

Em relação a causa do óbito, destaca-se o maior número de casos na causa de algumas afecções originadas no período perinatal, vale ressaltar a importância de uma assistência adequada durante todo esse período (tabela 2). 
Tabela 2: Percentual segundo a causa do óbito no capitulo CID - 10, ocorridos no Estado de Pernambuco no período de 2013 a 2017.

\begin{tabular}{lcc}
\hline Capitulo CID 10 & $\mathbf{N}^{*}$ & $\mathbf{\%}$ \\
\hline I. Algumas doenças infecciosas e parasitarias & 556 & 6,0 \\
IX. Doenças do aparelho circulatório & 35 & 0,4 \\
X. Doenças do aparelho respiratório & 397 & 4,3 \\
XVI. Algumas afecções originadas no período perinatal & 5.292 & 57,2 \\
XVII. Malf cong deformid e anomalias cromossômicas & 2.196 & 23,8 \\
Demais causas & 770 & 8,3 \\
Total & $\mathbf{9 . 2 4 6}$ & $\mathbf{1 0 0 , 0}$ \\
\hline
\end{tabular}

Fonte: MS/SVS/CGIAE - Sistema de Informações sobre Mortalidade - SIM / 20132017. *Número de Casos.

\section{DISCUSSÕES}

Os resultados desse estudo demonstram que o sexo masculino foi o mais frequente nos óbitos infantis no período de estudo no Estado de Pernambuco. O período neonatal precoce, mães em período etário de adultas jovens e escolaridade das mesmas acima dos oito anos também acompanhou esse resultado de predominância. Estudos realizados apontam que o perfil de mortalidade infantil, não divergem dos resultados desse estudo (FILHO, et al. 2017).

Foi observado o declínio de mortalidade nos anos analisados, porém se faz necessário evidenciar a intensificação de investimento em saúde no que tange à gestação, parto e nascimento, pois é notório que os óbitos neonatais precoces, possuem relação com a qualidade da assistência presta no pré-natal e neonatal durante o parto (MALTA, et al., 2019; LEAL, et al. 2017).

Em um estudo realizado sobre investigação de óbitos, identificou que as mortes infantis eram caracterizadas, em sua maioria, por crianças que não chegavam a receber alta após o nascimento. Evidenciou-se ainda a inadequação de informação de dados na assistência ao pré- natal e binômio. Sendo a investigação uma parte importante para identificação das falhas gerando ações na melhoria da 
assistência, é ideal observar durante a investigação as desigualdades na qualificação dos profissionais responsáveis por esta ação; a menor importância conferida durante o processo da coleta de dados; e o mau preenchimento de prontuários ambulatoriais e hospitalares (MALTA, et al., 2019; LEAL, et al. 2017; FILHO, et al. 2017).

Segundo o estudo de Leal (2017), destaca-se a invisibilidade das mulheres sob alto risco durante o acompanhamento no pré-natal, dado que $74,6 \%$ delas foram atendidas na atenção básica, sem serem referenciadas para serviços especializados. Esse percentual foi semelhante ao observado em mulheres classificadas como em risco obstétrico habitual 80,2\%. Dessa forma fica ainda mais obvio que são falhas na linha do cuidado da gestante, possivelmente pela ausência detecção e tratamento de condições adversas da gravidez (LEAL, et al. 2017).

O estudo de Migoto (2018), obteve como resultado a maior de chance de ocorrência de óbito neonatal precoce $50 \%$, aquelas que realizaram até seis consultas de pré-natal, quando comparadas as que realizaram sete ou mais. De acordo com a recomendação da Organização Mundial da Saúde, cada gestante deve realizar no mínimo uma consulta mensal até a vigésima oitava semana.

Nesse estudo, $57,2 \%$ dos óbitos infantis tiveram como causa afecções originadas no período perinatal. Essas causas são consideradas evitáveis, corroborando com estudos já realizados no município de Recife-PE e Teresina-PI. Estes óbitos poderiam ser prevenidos por ações de serviços de saúde acessíveis e afetivos, total ou parcialmente. Diante destes dados vale ressaltar mais uma vez a necessidade de melhorar o cuidado durante o pré-natal e o parto, de valorização do planejamento reprodutivo, do fortalecimento da atenção primária à saúde (LEAL, et al. 2017).

De acordo com a Organização Mundial de Saúde (OMS), para reduzir o número de casos perinatais, é importante atuação efetiva no pré-natal, no parto e na atenção ao recém-nascido. Houve uma ampliação da assistência pré-natal no país, o que é consistente com a ampliação da atenção básica e do Programa Saúde da Família.

Segundo Malta (2019), observou uma redução das causas por adequada atenção ao parto; causas como a hipóxia intrauterina, a asfixia ao nascer e a aspiração neonatal. A asfixia ao nascer e a hipóxia intrauterina são manifestações 
sindrômicas e a sua redução também pode ser decorrente da indicação mais correta de outras causas de morte. Investigação realizada no município de São Luís, capital do Maranhão, sobre todos os RN idos a óbito neonatal entre os anos de 2012 e 2014, encontrou como causas mais frequentes de óbito as respiratórias $(32,3 \%)$, sepse $(24,4 \%)$ e malformações congênitas (8,0\%).

Na pesquisa 'Nascer no Brasil', a prematuridade respondeu por cerca de um terço dos casos de óbitos neonatais, seguidos pelas malformações congênitas (23\%) e infecções (19\%).. A frequência das causas específicas de morte neonatal varia, entre contextos com diferentes taxas de mortalidade infantil; onde as principais causas de óbitos neonatais são prematuridade, asfixia ao nascer e causas congênitas (SZWARCWALD, 2014).

No estudo de Marques (2018), foi identificado uma redefinição da maioria das causas básicas do óbito infantil e a concordância das causas de óbito e da possibilidade de se evitar os casos, podendo esses dados serem considerados indicadores da adequação das ações da vigilância do óbito infantil. O registro inadequado de causas intermediárias, em desvantagem de causas específicas, pouco contribui para a compreensão das condições de mortalidade; o que também compromete a identificação dos fatores de risco ao recém-nascido, possivelmente relacionados a problemas intrauterinos, maternos, placentários ou do próprio feto.

Frente ao exposto a prevenção oportuna de agravos durante o processo gestacional seja possível pelo monitoramento e acompanhamento da mulher a partir da suspeita ou confirmação do processo gestacional, o qual pode ser favorecido pelo planejamento e busca ativa pela atenção primária. É reconhecido que a cobertura da assistência ao pré-natal e ao parto é quase universal no Brasil (LEAL, et al. 2017).

\section{CONCLUSÃO}

Esse estudo demonstrou o declínio de óbitos infantis durante o período que foi analisado. O resultado do perfil dos casos não divergem dos estudos que apontam o perfil materno e dos óbitos infantil, como também das causas dos óbitos 
ter sido perinatais, ou seja, está relacionado ao pré-natal, parto e nascimento. Assim, como observado nos estudos anteriores, seus achados, achados revelam a necessidade de melhorar a assistência com o intuito de prevenir esses fatores que são os principais responsáveis pelos altos coeficientes de mortalidade neonatal.

Vale ressaltar que a maioria das causas dos óbitos são evitáveis, com tudo é importante avaliar a resolutividade dos serviços de saúde materno-infantil, além de fornecer estimativas que auxiliam na tomada de decisões e no planejamento de políticas públicas.

Acredita-se que para melhor redução das taxas de mortalidade infantil, é necessária uma ampliação na especialização dos profissionais que atuam diariamente na área de pré-natal, parto e nascimento, com materno-infantil. Visto que para evitar a maior parte da mortalidade é importante que se tenha qualidade e uma atenção adequada ao parto e ao recém-nascido.

\section{REFERÊNCIAS BIBLIOGRÁFICAS}

Departamento de Informático do Sistema Único de Saúde (DATASUS). FILHO A. SALES I. ARAÚJO A. ALMEIDA P. ROCHA S. Aspectos epidemiológicos da mortalidade neonatal em capital do nordeste do Brasil. Rev Cuid 2017; 8(3): 1767-76.

OLIVEIRA C. GUIMARÃES M. FRIAS P. BONFIM C. ANTONINO V. GUIMARÃES L. MEDEIROS Z. Adequação da investigação dos óbitos infantis no Recife, Pernambuco. Brasil; Ciência \& Saúde coletiva 23 (3):701-714,2018.

MIGOTO M. OLIVEIRA R. SILVA A. FREIRE M. Early neonatal mortality and risk factors: a case-control study in Paraná State. Rev Bras Enferm. 2018;71(5):2527-34.

MALTA C. PRADO R. SALTARELER M. MONTEIRO R. SOUZA M.F. ALMEIDA M. Mortes evitáveis na infância, segundo ações do Sistema Único de Saúde. Brasil; rev bras epidemiol 2019; 22: e190014.

MATTO A. CACCIA-BAVAI M.C. BARBOSA D. Índice de Saúde Aplicado ao Município de Araraquara, SP: um instrumento para o acompanhamento da Atenção Básica. Rev Bras Epidemiol 2013; 16(1): 210-22.

LEAL M. BITTENCOURT S. TORRES R. NIQUINI R. JUNIOR P. Determinantes do óbito infantil no Vale do Jequitinhonha e nas regiões Norte e Nordeste do Brasil. Rev Saúde Pública 2017;51:12.

SZWARCWALD, C. FRIAS, P. JUNIOR, P. ALMEIDA, W. NETO, L. Correction of vital statistics based on a proactive search of deaths and live births: evidence from a study of the North and Northeast regions of Brazil. Popul Health Metr. 2014 Jun; 12: 16.

MARQUES, L. PIMENTEL, D. OLIVEIRA, M. VILELA, M. FRIAS, P. BONFIM C. Concordância 
da causa básica e da evitabilidade dos óbitos infantis antes e após a investigação no Recife, Pernambuco, 2014. Epidemiol. Serv. Saude, Brasília, 27(1): e20170557, 2018.

TEIXEIRA J. ARAUJO W. MARANHÃO A. ESCALANTE J. REZENDE L. MATIJASEVICH A. Mortalidade no primeiro dia de vida: tendências, causas de óbito e evitabilidade em oito Unidades da Federação brasileira, entre 2010 e 2015. Epidemiol. Serv. Saude, Brasília, 28(1):e2018132, 2019.

PADOVANI C; OLIVEIRA R.; PELLOSO S.; Sífilis na gestação: Associação das características maternas e perinatais em região do sul do Brasil. Rev. Latino-Am. Enfermagem 2018.

OLIVEIRA E. PEZZATO L. MENDES R. Às margens do cuidado: regulações de gênero em uma equipe de saúde. Revista de Saúde Coletiva, Rio de Janeiro, V. 28(2), 2018.

MATTOS A. CACCIA-BAVA M.C. BARBOSA D. Índice de Saúde Aplicado ao Município de Araraquara, SP: um instrumento para o acompanhamento da Atenção Básica. Rev. bras. epidemiol. 16 (1) Mar 2013.

SANTOS H. ANDRADE S. SILVA A. CARVALHO W. MESAS A. GONZÁLEZ A. Concordância sobre causas básicas de morte infantil entre registros originais e após investigação: análise de dois biênios nos anos 2000. Rev Bras Epidemiol abr-jun 2014; 313- 322.

TEIXEIRA J. ARAUJO W. MARANHÃO A. CORTEZ-ESCALANTE J. REZENDE L. MATIJASEVICH A. Mortalidade no primeiro dia de vida: tendências, causas de óbito e evitabilidade em oito Unidades da Federação brasileira, entre 2010 e 2015. Epidemiol. Serv. Saude, Brasília, 28 (1): e2018132, 2019. 